\title{
DISEÑO DE UNA UNIDAD DIDÁCTICA COMO ESTRATEGIA PARA ABORDAR LA ENSEÑANZA DE LA HERENCIA MENDELIANA EN ESTUDIANTES DE GRADO NOVENO
}

\section{Design of a unit as a strategy to address the teaching of mendelian inheritance ninth grade students}

Fecha de recepción: 24 de marzo de 2014

Fecha de aprobación: 10 de diciembre de 2014

Fecha de aprobación: 10 de diciembre de 2014

\author{
María Camila Hurtado Torres ${ }^{1}$ \\ Mayra Alejandra Cañizares Martínez ${ }^{2}$
}

\section{Resumen}

En este artículo se fundamenta teóricamente una unidad didáctica, dirigida a estudiantes de grado noveno, relacionada con el concepto de herencia mendeliana, en la que se busca abordar la enseñanza de la genética de una manera más dinámica, ya que esta ciencia se considera básica para la comprensión de muchos de los fenómenos biológicos, para lo cual se plantearon tres objetivos: a) lograr por medio de una unidad didáctica la comprensión de conceptos como: homocigoto, heterocigoto, dominante, recesivo, alelo, gen; b) promover el aprendizaje por medio de resolución de situaciones problémicas que le permitan aplicar tanto los conceptos biológicos como algunas funciones básicas de la matemática, y c) facilitar en los alumnos el reconocimiento de los diferentes caracteres genéticos, no solo en cuanto a enfermedades sino en cuanto a algunas características morfológicas de la diversidad en la tierra. Para llevarlo a cabo, se decidió utilizar el enfoque de investigación escolar, propuesto por García y Merchán (1997), quienes consideran importante la inclusión del contexto y de los imaginarios de los alumnos para lograr la construcción de un conocimiento escolar. Esta investigación se desarrolló durante siete sesiones en las cuales se encontró que la mayoría de los estudiantes lograron una apropiación del conocimiento debido a que la unidad además de contemplar el contexto social y el territorio, maneja el aprendizaje basado en la acción o cooperación (ABA), lo cual les permite despertar sus conocimientos y emociones a través la indagación y divulgación con sus compañeros.

\section{Palabras clave}

Herencia mendeliana, contexto social, investigación escolar, aprendizaje basado en la acción (ABA), especie.

\section{Abstract}

In this paper the theoretical foundations of a teaching unit aimed at ninth grade students, related to the concept of Mendelian inheritance, which seeks address the teaching of genetics in a more dynamic way., as this is considered basic science for understanding many biological phenomena, for it raised to 3 targets) achieved through a teaching unit understanding of concepts such as: homozygous, heterozygous, dominant, recessive allele, gene; b) promote learning through solving problematic situations that allow you to apply both biological concepts as some basic functions of mathematics and c) allow students to recognize different genetic traits, not only in terms of illness but as to morphological aspects of diversity on earth. To carry it out, it was decided to use the proposed by Garcia in 1997 and considers it important to include the context and imaginary students to achieve the construction of a school knowledge school research approach. This research was conducted for 7 sessions in which it was found that most students made an appropriation of knowledge because the unit in addition to providing the social context and territory manages learning based on action or cooperation (ABA), which allows them to wake their knowledge and emotions through inquiry and disclosure with peers.

\section{Key words}

Mendelian inheritance, social context, school research, learning based on action (ABA), specie

\footnotetext{
1 Licenciada en Biología. Universidad Distrital Francisco José de Caldas. Correo electrónico: mchurtadot@gmail.com

2 Licenciada en Biología. Universidad Distrital Francisco José de Caldas. Correo electrónico: alejasally@gmail.com
} 


\section{Introducción}

Se desarrolló una unidad didáctica en grado noveno, en los cursos 902 y 903 del Colegio Ateneo Juan Eudes, ubicado en el barrio Minuto de Dios, localidad de Engativá, donde los estratos de los alumnos oscilan entre 3 y 6 . Esta se encaminó hacia un proceso de enseñanza y aprendizaje enfocado en el contenido de la genética mendeliana. Teniendo en cuenta lo anterior, los contenidos temáticos a se situaron en el contexto cotidiano de los estudiantes, para lo cual se utilizó la propuesta de García y Merchán (1997), que hace alusión a la equivalencia entre lo cotidiano y lo científico, sin necesidad de realizar reestructuraciones fuertes para pasar de una a otra.

Los conceptos se organizaron teniendo en cuenta el enfoque del programa de nivelación, recuperación del año perdido y avance de grado simultáneo de la institución Ateneo Juan Eudes, el Proyecto Educativo Institucional (PEI), el grado de desarrollo de los alumnos y los objetivos planteados para la investigación, los cuales se encaminaron principalmente a promover el interés de los estudiantes hacia la genética, ya que esta ciencia se considera básica para la comprensión de muchos de los fenómenos biológicos (Benítez, 2013). Para lograr esto se plantearon tres objetivos específicos que, en primera instancia, buscan que el estudiante comprenda y aplique conceptos como: homocigoto, heterocigoto, dominante, recesivo, alelo, gen; ya que el entendimiento de estos le permitirá fortalecer su entendimiento acerca de las leyes de Mendel; en segunda medida se buscó promover en el alumno la resolución de situaciones por medio de planteamientos de problemas que le permitan no solo aplicar los conceptos biológicos, sino también algunas funciones básicas de la matemática, y en última medida se pretendía que los estudiantes reconocieran los diferentes caracteres genéticos, no solo en cuanto a enfermedades sino a las características morfológicas de algunos individuos pertenecientes a su ambiente. De igual manera, el planteamiento de la metodología empleada se encaminó hacia el enfoque de investigación escolar (García y Merchán, 1997), el cual se basa en el planteamiento y solución de problemas a lo largo de una secuencia de enseñanza/aprendizaje.

La genética mendeliana -aspecto trabajado- es considerada como un tema esencial en la formación básica estudiantil, ya que según Benítez (2013), e liñiguez y Puigcerver (2013) le permitirá al alumno identificar la influencia del ambiente en su genoma y, por tanto, valorar la repercusión social de temáticas tan determinantes como la ingeniería genética, la terapia génica, la clonación y otras manipulaciones en el material hereditario que sin duda afectan tanto a la naturaleza como al ser humano. Ade- más, el conocimiento acerca de la herencia de los seres vivos facilitará en el estudiante una idea de cómo se establecieron algunos de los organismos que ellos observan cotidianamente.

Para implementar esta temática se eligió el grado noveno, ya que en esta etapa los estudiantes comprenden conceptos relacionados con la organización de los seres vivos, estructura y función celular, y la reproducción, con los cuales pueden identificar la importancia de los organismos y las diferentes interacciones entre ellos.

En esta unidad didáctica se buscó indagar en primera medida la relación que tiene la genética con el desarrollo de la vida y el entorno de los alumnos. Además de presentar un escenario interactivo en el cual se comprendieran a través de la resolución de problemas la influencia del ambiente en el funcionamiento de los individuos. Para ello, inicialmente se llevó a cabo una indagación a los conceptos previos de los educandos (preconceptos/conceptos) para así determinar el conocimiento conceptual y contextual de ellos (Rayas, 2004). Posterior a esto se llevaron a cabo actividades y talleres para el reconocimiento de algunos caracteres presentes en los individuos de muestra y de cómo el cruce entre estos puede generar la diversidad entre las especies.

\section{Materiales y métodos}

Esta unidad se llevó a cabo en el Colegio Ateneo Juan Eudes, ubicado en la transversal 74 N.o 81C-05, en la zona 10 de la localidad de Engativá, en el barrio Minuto de Dios. Esta institución maneja estudiantes de estratos 3 a 6 y ofrece el programa de nivelación, recuperación del año perdido y avance de grado simultáneo, diseñado para estudiantes promovidos con áreas perdidas o años reprobados. El rango de edad de los estudiantes es de 13 a 20 años y los grados que ofrece la institución van desde séptimo hasta undécimo.

El Proyecto Educativo Institucional (PEI) del Colegio Ateneo Juan Eudes es "Educación integral humana con sentido y calidad por medio del amor, servicio, justicia, libertad y honradez", el cual permitió emplear el enfoque de investigación escolar que plantea la solución de problemas a lo largo de una secuencia de enseñanza/aprendizaje, donde la exploración del alumno se identifica con las diversas formas de conocimiento presentes en la actividad humana, más específicamente investigaciones de situaciones problemáticas, que incluyen a nuestra comunidad, a los demás seres vivos y al medio que los rodea. Esta metodología que genera situaciones novedosas para el estudiante pretende que él proponga soluciones o elabore respuestas para la construcción de un nuevo conocimiento. 
De acuerdo con García y Merchán (1997), el nuevo conocimiento producto de este enfoque se denomina conocimiento escolar, que se caracteriza por estar organizado en un sistema de ideas y cuya construcción se da de manera gradual, siendo comparado con los niveles de formulación, que son el conjunto de conocimientos que sirven como marco de referencia (conocimiento escolar deseable), guiando la organización y secuenciación de los contenidos, pero también pueden hacer alusión a la compresión que manifiestan los alumnos al explicitar las ideas. Para ello se deben tener en cuenta elementos como la significancia de los contenidos, la relación entre lo cotidiano y lo científico, la complejidad de lo enseñado y el resultado del aprendizaje, pues tener esto claro nos va a permitir alcanzar un apropiación conjunta del conocimiento (Carmen, 1997)

Lo interesante de este enfoque es que tiene en cuenta al individuo, ya que a partir de sus vivencias y de su experiencia puede resolver problemáticas y comprender, con ayuda del docente, diversos temas presentes en su entorno, que en este caso fue la genética mendeliana, considerada como un concepto fundamental para entender la biología.

Para el desarrollo de este proyecto se llevaron a cabo siete actividades: la primera se basó en la caracterización del docente, en la cual se observaron y analizaron tópicos relacionados con el manejo de la temática, el dominio del curso y la relación docente/estudiante. De la misma manera se buscó realizar una caracterización socioeconómica y académica de la población estudiantil, aplicando un formato de ideas previas (anexo 1).

Posteriormente, a partir de la información establecida en las caracterizaciones previas se diseñaron e implementaron unas actividades durante la tercera sesión para que los estudiantes reconocieran conceptos de fenotipo, genotipo y gen desde los cruces hipotéticos de organismos como amblipigios, uropígidos, ranas, grillos, escorpiones, coleópteros y diplópodos (anexo 2). Al inicio de la actividad se explicó la morfología de cada uno de los organismos, con los ejemplares preservados como material de estudio por la Universidad Distrital; individuos que forman parte de la colección del Museo UDFJC. Con estos instrumentos de apoyo los alumnos se organizaron en grupos para reconocer las diferencias en cada uno de los especímenes, como actividad de conceptualización del fenotipo. A partir de esta actividad, se plantearon situaciones problemas como la siguiente: "Un coleóptero macho con cuerno largo dominante se cruza con una hembra de cuerno corto recesivo. De este cruce determine el genotipo y el fenotipo". Al finalizar esta sesión se les asignó como trabajo la consulta del individuo que les correspondió teniendo en cuenta aspectos como su importancia ecológica, su clasificación taxonómica, su hábitat y sus características principales.

\section{Ejemplos de caracteres dominantes y recesivos en los seres humanos}

En la siguiente clase, cada grupo expuso frente a sus compañeros el organismo trabajado, con el propósito de evidenciar las características identificadas y la importancia que lograron establecer de cada uno de ellos en su entorno; posteriormente, fueron evaluados por sus compañeros y los docentes a cargo, teniendo en cuenta criterios como la presentación del trabajo y el dominio del tema. Como parte de la actividad se realizó una retroalimentación con el fin de establecer una adecuada consolidación de las temáticas trabajadas durante esta sesión.

Para la quinta actividad, inicialmente se realizó la explicación de algunos caracteres dominantes y recesivos que se pueden encontrar en los seres humanos y cómo estos se evidencian en el genotipo y fenotipo, resaltando los caracteres morfológicos en ellos mismos, para así llevar a cabo una evaluación más contextualizada (anexo 3), en la cual se propuso la resolución de diversos problemas, como por ejemplo: "Un hombre con alelos dominantes para la característica de poder enrollar la lengua, se casa con una mujer que no puede hacerlo. Los alelos están representados por las letras 'LL' para el dominante y 'Il' para el recesivo. ¿Qué fenotipo y genotipo se esperaría de esta unión en su descendencia?". Dentro de la información presentada para la resolución de estos problemas, se colocó como guía un cuadro de características dominantes y recesivas de los seres humanos (tabla 1). Se finalizó con una pequeña consulta sobre cruces dihíbridos y la elaboración de un ejemplo. 


\begin{tabular}{|c|c|c|c|}
\hline \multicolumn{2}{|c|}{ CARACTERES TRIVIALES } & DOMINANTES & Enanismo \\
DOMINANTES & RECESIVOS & Estatura normal \\
\hline Lengua enrollable & Lengua no enrollable & Braquidactilia & Dedos normales \\
\hline Rh+ & Rh - & Corea de Huntington & Sin Corea de Huntington \\
\hline Pelo rizado & Pelo liso & Pigmentación normal & Albinismo \\
\hline Cabello oscuro & Cabello claro & Coagulación normal de la sangre & Hemofilia \\
\hline Ojos oscuros & Ojos claros & Visión normal & Daltonismo \\
\hline Labios gruesos & Labios finos & Oído normal & Sordomudez \\
\hline Pestañas largas & Oreja sin lóbulo & Polidactilia & N. ${ }^{\circ}$ normal de dedos normal \\
\hline Oreja con lóbulo & Grupo sanguíneo O & Ceguera para los colores \\
\hline
\end{tabular}

Tabla 1. Ejemplos de caracteres dominantes y recesivos en seres humanos Tomada de: http://www.quimicaweb.net/Weblumnos/GENETICA\%20Y\%20HERENCIA/Paginas/8.htm

En la siguiente sesión se elaboró una construcción conjunta con los alumnos, del concepto de cruces dihíbridos, partiendo de su consulta previa y algunos ejercicios desarrollados por las docentes a cargo; posteriormente con el objetivo de profundizar más en esta temática y lograr una mejor comprensión, se organizaron a los estudiantes en grupos de cuatro integrantes para desarrollar problemas en los cuales se tienen en cuenta dos caracteres de los organismos trabajados en la tercera sesión.

Ya en la última sesión se implementó una evaluación que contenía ejercicios dihíbridos, de diversos individuos como seres humanos, animales y plantas (anexo 4), con preguntas como: "Un caballo macho de crin largo (dominante, LL) y pelaje negro (dominante, NN) se cruza con una yegua de crin corto (recesivo, II) y pelaje blanco (recesivo, nn). A partir de esto determinar el genotipo y el fenotipo para su descendencia". Al culminar esta evaluación los problemas que presentaban algunos errores en su desarrollo, se corrigieron con la participación tanto de los estudiantes como de los docentes a cargo, resolviendo algunas dudas o inquietudes de los alumnos, para lograr así una mejor comprensión.

Con cada una de estas actividades, se buscó que los estudiantes reconocieran las razones por las cuales existe tanta diversidad entre las especies, además del factor genético obtenido del intercambio de material heredi- tario, teniendo en cuenta, claro está, la influencia del ambiente. También se pretendió generar en ellos una conciencia de apropiación por cada uno de los seres vivos que habitan en su entorno, ya que resulta importante el reconocimiento de su medio y la variabilidad que existe.

\section{Resultados y discusión}

Por medio de la caracterización docente se logró observar que el desarrollo de la clase se da bajo las herramientas tomadas del enfoque expositivo y algunas propuestas de Pozo (2006)8; ya que determinamos que la explicación de los conceptos básicos de la genética mendeliana, expuestos durante su cátedra, buscaban una interacción continua entre los estudiantes y su propia concepción a la hora de la construcción de los conceptos a trabajar. Esto con la finalidad de lograr una compatibilidad entre los conocimientos científicos y los cotidianos de los propios estudiantes (Pozo 1998). Dicha estrategia coincide con la propuesta establecida por Ausubel (1973), quien considera "no solo la lógica de la disciplina, sino también la lógica de los alumnos, teniendo en cuenta que el aprendizaje de la ciencia consiste en transformar el significado lógico en significado psicológico".

Para la siguiente actividad se implementó el instrumento de caracterización estudiantil que suministró información de aspectos personales y socioeconómicos de cada estu- 
diante, con el fin de contextualizar sus intereses y recursos. En los resultados podemos establecer inicialmente que la mayoría de los estudiantes se encuentran en el estrato 4 (gráfico 1) y que su rango de edad oscila principalmente entre los 14 y 15 años (gráfico 2).

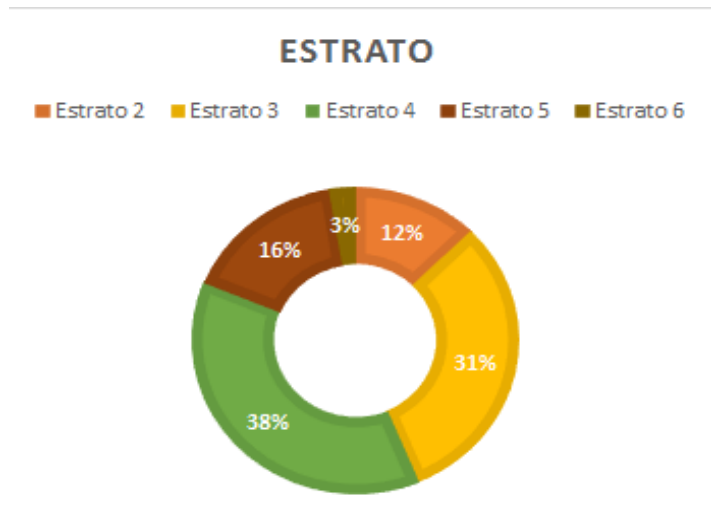

Gráfico 1. Estratos de los estudiantes de los cursos 902-903 del Ateneo Juan Eudes.

\section{EDADES}

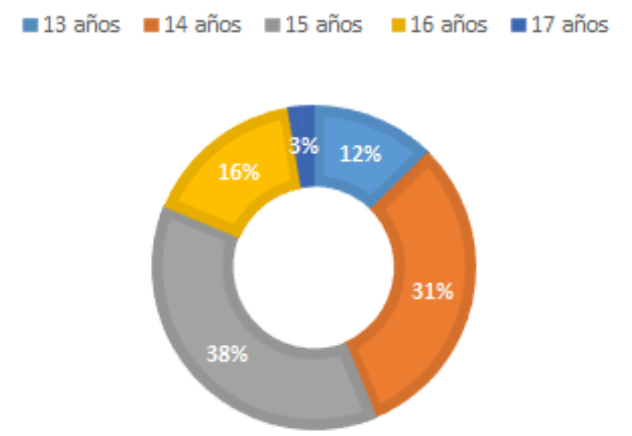

Gráfico 2. Rangos de edades de los alumnos de los cursos 902-903 del Ateneo Juan Eudes.

Además de esto se puede establecer que los estudiantes no prestan gran interés por las Ciencias Naturales (gráfico 3), debido a que en muchos casos se utilizan las mismas metodologías de enseñanza, las cuales en gran medida resultan repetitivas y aburridas, se basan en las mismas guías de laboratorio y en la explicación magistral (Miguens y Garret, 1991).

\section{CUAL ES LA ASIGNATURA QUE MAS TE GUSTA EN TU COLEGIO}
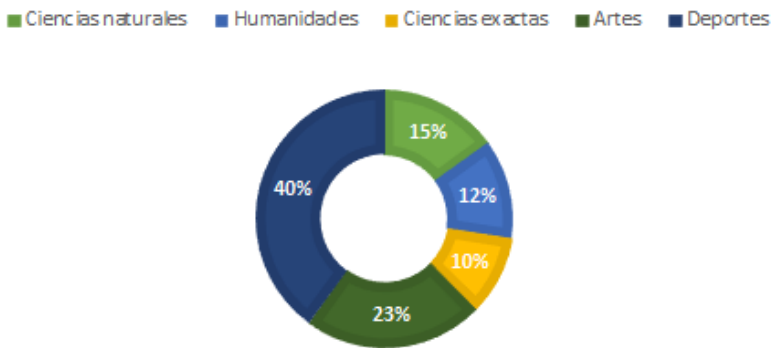

Gráfico 3. Preferencias de asignaturas de los estudiantes de los cursos 902-903 del Ateneo Juan Eudes.

Para sus trabajos, en la mayoría de los casos, los alumnos utilizan Internet (gráfico 4), herramienta que les proporciona una facilidad en la búsqueda y organización de la información; de hecho, la gran mayoría manifestó que sería muy bueno introducir plataformas virtuales para el manejo de las clases y la realización de tareas. Esto lo respalda Pósito (2012), quien asegura que así se puede llegar a complementar el trabajo del aula real.

\section{CUÁLES HERRAMIENTAS UTILIZA PARA REALIZAR SUS TAREAS}

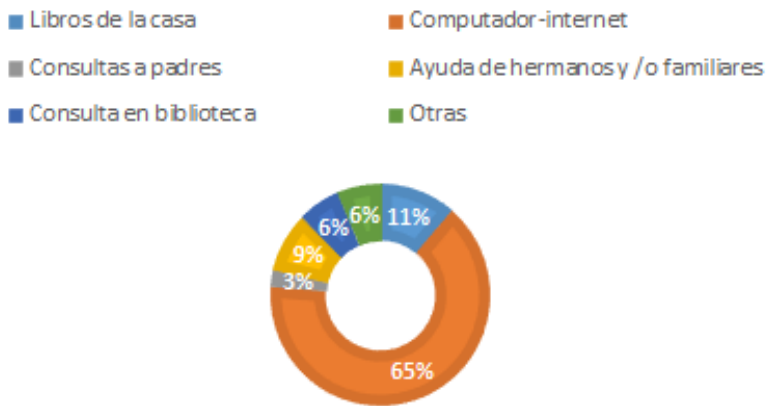

Gráfico 4. Principales herramientas utilizadas por los alumnos de 902-903 del Ateneo Juan Eudes para la realización de trabajos

Además podemos establecer que a partir de las preguntas realizadas, con relación a los conceptos previos, los alumnos no reconocen las características observables como fenotipo, ni tienen conocimientos sobre el significado de genotipo, también es evidente que no identifican la herencia y el ambiente como constituyente principal de la gran biodiversidad y del factor evolutivo. 
Entre sus concepciones se evidencia que la mayoría se considera como la especie más desarrollada, en la cual el resto de los organismos esta en pro de su bienestar, mas no que el ser humano es un fragmento de un todo. Los estudiantes tampoco saben la diferencia taxonómica de los individuos ni logran identificar especies representativas de cada orden. Esta percepción está fundamentada en las respuestas de los estudiantes a las preguntas relacionadas con las diferencias anatómicas de los seres vivos, clasificación de los mismos e importancia y trascendencia de los seres humanos en el ambiente. Por ejemplo podemos citar respuestas como: "Somos los seres superiores y por eso los animales nos sirven a nosotros, porque son menos desarrollados" y "Dentro de los equinodermos podemos encontrar a los caballos". Además de esto podemos evidenciar que la mayoría de los alumnos presentan vacíos conceptuales en temáticas relacionadas con la organización de los seres vivos, ya que al solicitarles que ejemplificaran un individuo por cada Phylum, ellos no lograron reconocerlos y tan solo podían hacer referencia a los vertebrados. Resaltando que la organización evolutiva de los organismos permite generar una caracterización morfológica específica de cada especie y cuáles caracteres han permanecido dominantes hasta la actualidad.

A partir de estas ideas previas se direccionaron las actividades para aclarar los conceptos y vacíos, ya que según Gil (1993), esto permite en primer lugar, poseer un conocimiento acerca de las concepciones con las que los estudiantes enfrentan el aprendizaje de los conocimientos científicos. En segundo lugar ha puesto de manifiesto que dicho aprendizaje lleva incorporado un interesante problema de transformación conceptual. Por último, la existencia de las ideas previas pone de manifiesto el desafío de enfrentar los procesos de aprendizaje, bajo una óptica distinta, puesto que para producir el cambio conceptual no bastaría solo con tomar en consideración estas ideas previas, sino también que esto debe ir acompañado de una metodología de enseñanza que incorpore los interrogantes de los estudiantes y sus propuestas.

En la tercera actividad los resultados fueron muy favorables, ya que los estudiantes tuvieron más iniciativa y mostraron interés en la apropiación del conocimiento relacionado con la genética mendeliana, gracias al material empleado en este ejercicio, que permitió partir del reconocimiento de un organismo tangible. Los alumnos al tener la posibilidad de observar las características fenotípicas y asociar a su vez los conceptos morfológicos de los individuos, les permiten tener una mayor asociación conceptual con los términos trabajados. La resolución de problemas de esta actividad fue adecuada, ya que observamos que dentro de las exposiciones del trabajo grupal sobre los cruces monohíbridos se evidenciaron respuestas más acertadas. Esta metodología arrojó buenos resultados, ya que está basada en la propuesta de Guitart (2011), quien establece el entendimiento por ABA (aprendizaje basado en la acción) como una estrategia o metodología de enseñanza y aprendizaje que se basa en el trabajo cooperativo alrededor de actividades significativas, que logran una adecuada mediación instrumental y social y una vinculación intelectual, emocional y territorial. Además propone:

Los procesos de enseñanza y aprendizaje se generan a través del trabajo colaborativo, guiado por el profesor o la profesora, alrededor de la combinación de situaciones contextualizadas (fuera del salón de clase) y descontextualizadas (en el salón de clase).

Posteriormente, cada uno de los grupos previamente trabajados presentó una exposición del animal asignado anteriormente, mostrando aspectos como su hábitat, sus características morfológicas y su importancia ambiental (imagen 1), como material de apoyo para esta actividad elaboraron un dibujo de cada especie con el fin de mostrarles a sus compañeros la influencia del ambiente sobre las características observadas. Al transcurrir la actividad se evidenció la comprensión entre la relación del genotipo, el fenotipo con el ecosistema, ya que cada grupo elaboró, a partir de la consulta, su propia comprensión de la temática trabajada, y expuso este tipo de interacción. Por medio de este ejercicio se pudo observar cómo los estudiantes interiorizan los nuevos conocimientos para posteriormente transmitirlos y explicarlos a sus demás compañeros, con lo que se demostró el verdadero significado del aprendizaje, de igual manera lograron identificar los organismos que los rodea y su importancia biológica.

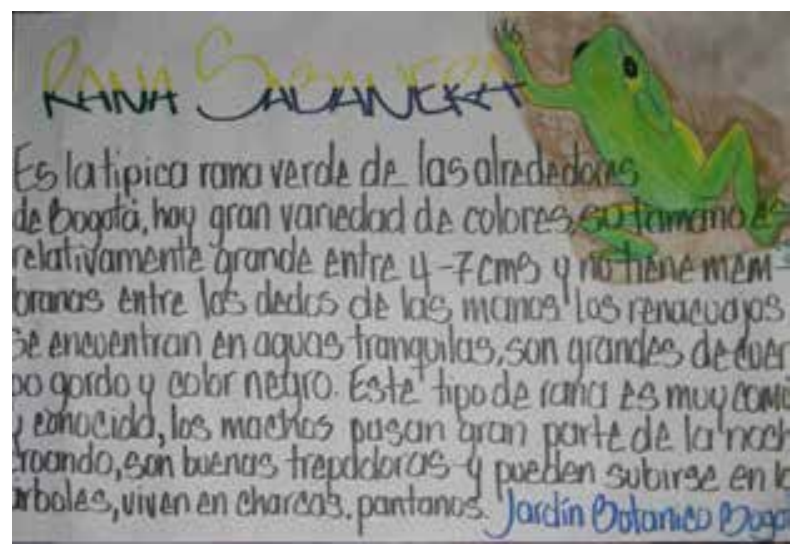

Imagen 1. Cartelera sobre el organismo trabajado en la sesión 4. 
Al finalizar el proceso de construcción conceptual de los cruces monohíbridos, se llevó a cabo una evaluación, donde obtuvimos los resultados ilustrados en los gráficos 5 y 6 .

\section{Resultados del primer examen de monohíbridos curso 902}

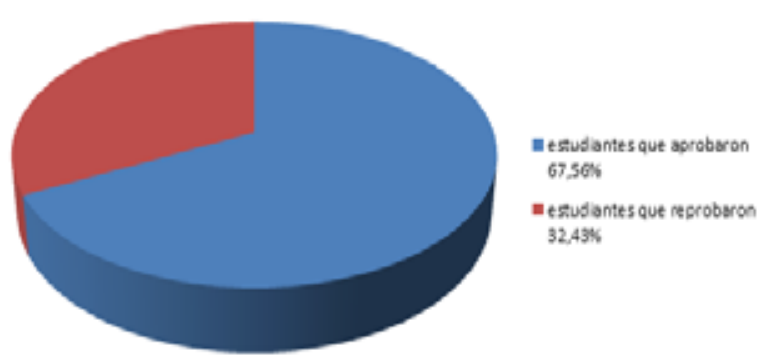

Gráfico 5. Resultados del primer examen realizado al curso 902

\section{Resultados del primer examen de monohíbridos curso 903}

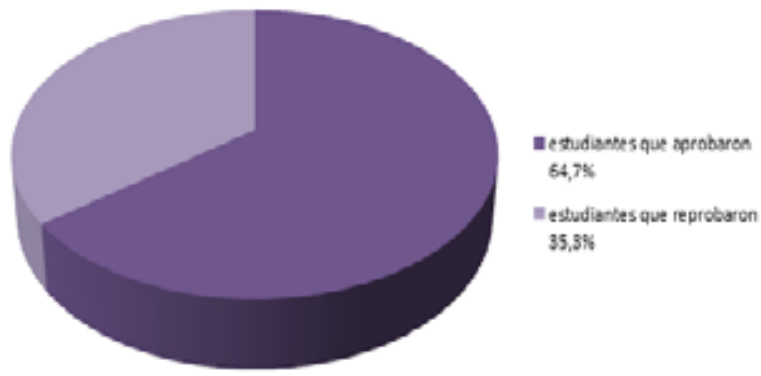

Gráfico 6. Resultados del primer examen realizado al curso 903

Con estos resultados podemos establecer que las metodologías trabajadas arrojaron un buen rendimiento, ya que más de la mitad del curso logró una adecuada comprensión de las temáticas trabajadas.

Ya en la sexta clase, junto con los estudiantes, se definió el concepto de dihíbrido, estableciendo sus principales características y su diferencia con los cruces sencillos; conjuntamente se realizaron diversos ejercicios para así proceder a resolver, en grupos de cuatro personas, un problema relacionado con esta temática. De esta actividad se obtuvieron muy buenos resultados, ya que al trabajar las características fenotípicas de los mismos ejemplares fue más sencillo para ellos establecer vínculos entre estas, todo lo anterior fue evidente porque a la hora de resolver los problemas los estudiantes generaron aportes significativos y hubo mayor participación.
En la última sesión se llevó a cabo una evaluación de los cruces dihíbridos, en la cual se obtuvieron los resultados que se muestran en los gráficos 7 y 8 .

\section{Resultados del segundo examen de dihíbridos curso 902}

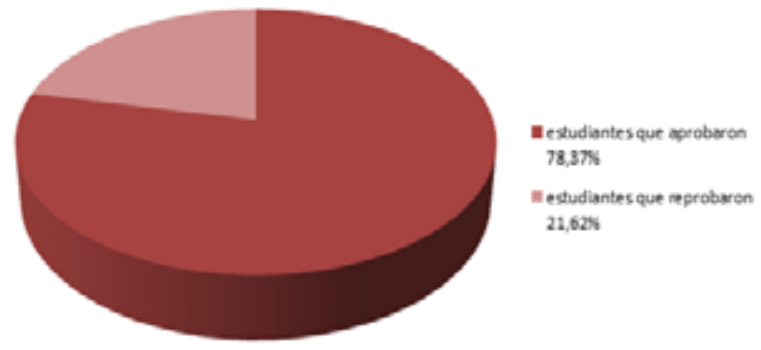

Gráfico 7. Resultados del segundo examen realizado al curso 902

\section{Resultados del segundo examen de dihíbridos curso 903}

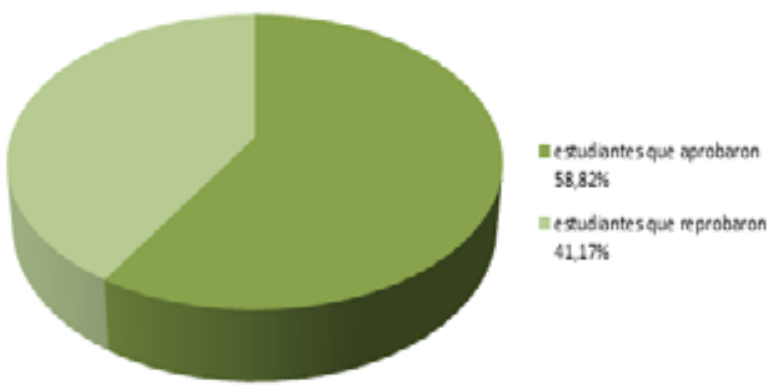

Gráfico 8. Resultados del segundo examen realizado al curso 903

De acuerdo con lo anterior podemos constatar que más de la mitad del curso obtuvo un buen resultado y comprendió la temática, pues además de dar respuesta a cada uno de los problemas planteados se apropiaron de un lenguaje científico al utilizar y entender cada uno de los términos trabajados durante la unidad.

Con todos los resultados durante este proceso, es evidente que se puede hacer uso de herramientas que permitan una conceptualización a través de la experiencia y del contexto en el que se desenvuelven los estudiantes, ya que enfrentar a estos de cara a una realidad inmediata genera en ellos un interés hacia el aprendizaje, además es un recurso práctico para el docente pues le permite entrelazar la teoría con la realidad y generar una conexión de los estudiantes con su entorno. 
Por último, se pudo constatar que el concepto de genética mendeliana en realidad se puede considerar como base, pues en este caso nos permitió explicar temáticas como la taxonomía y la ecología de cada uno de los organismos trabajados, al relacionarlo con el fenotipo y el genotipo.

La metodología implementada en esta unidad didáctica (especies tangibles) despertó la curiosidad de los estudiantes al demostrar su interés, manifestándose en la profundización en estos organismos, de tal manera que emplearían parte de su tiempo en buscar información relacionada con los individuos trabajados, ya que estos a pesar de encontrarse en su entorno resultan enigmáticos para ellos.

\section{Conclusiones}

Por medio de las actividades realizadas en el Ateneo Juan Eudes se logró despertar el interés en los estudiantes de noveno grado, hacia los conceptos trabajados y, en especial, hacia los organismos presentados.

Al observar un promedio de los resultados de los exámenes realizados, se pudo concluir que el enriquecimiento de las actividades fue amplio y permitió una comprensión adecuada por parte de los estudiantes hacia las temáticas trabajadas.

La implementación de esta unidad didáctica como puente importante de comprensión conceptual de la herencia es de gran ayuda para futuras aplicaciones en esta institución, ya que arrojó muy buenos resultados en los estudiantes.

Con las actividades realizadas en el grado noveno, se observó que los estudiantes comenzaron a tener procesos de relación conceptual con su diario vivir, al relacionar su ambiente con el de estos organismos.

Gracias a las actividades y el material implementado los estudiantes comprendieron conceptos como homocigoto, heterocigoto, dominante, recesivo, genotipo, fenotipo, alelo, gen y cálculos porcentuales de los cuadros de Punnet.

\section{Referencias bibliográficas}

Ausubel, B. (1973). Algunos aspectos psicológicos de la estructura del conocimiento. En: S. Elam (comp.). La educación y la estructura del conocimiento. Investigaciones sobre el proceso de aprendizaje y la naturaleza de las disciplinas que integran el currículo (pp. 211-239). Buenos Aires: El Ateneo.

Banet, E. (2000). La enseñanza y el aprendizaje del conocimiento biológico. En: F. Palacios y P. León (eds.). Didáctica de las ciencias experimentales (pp. 449478). España: Marfil.
Benítez, R. (2013). La enseñanza de la genética en el grado noveno de básica secundaria: una propuesta didáctica a la luz del constructivismo. Tesis de grado no publicada, para optar por el título de Magíster en Enseñanza de las Ciencias Exactas y Naturales. Medellín: Universidad Nacional de Colombia.

Carmen, L. (1997). El conocimiento escolar: ¿interesante, útil, deseable o posible? Revista Investigación en la Escuela, (32), 63.

García, J. y Merchán, F. (1997). El debate de la interdisciplinariedad en la E.S.O.: el referente metadisciplinar en la determinación del conocimiento escolar. Revista Investigación en la Escuela, (32), 5-26.

Gil, P. (1993). Propuestas alternativas para la introducción de los conceptos científicos: de aprendizaje como cambio conceptual al aprendizaje como investigación. Valencia: Editorial Popular.

Guitart, M. (2011), Del "aprendizaje basado en problemas" (ABP) al "aprendizaje basado en la acción" (ABA). Claves para su complementariedad e implementación. Revista de Docencia Universitaria 9(1), 91-107.

Íñiguez, F. y Puigcerver, M. (2013). Una propuesta didáctica para la enseñanza de la genética en la educación secundaria. Revista Eureka sobre Enseñanza y Divulgación de las Ciencias, 10(3), 307-327.

Miguens, M. y Garrett, R. (1991). Prácticas en la enseñanza de las ciencias: Problemas y posibilidades. Revista Investigación y experiencias didácticas 9(3), 229-236.

Pósito, M. (2012). El problema de enseñar y aprender Ciencias Naturales en los nuevos ambientes educativos: diseño de un gestor de prácticas de aprendizaje GPA. Trabajo de tesis realizado como requisito para optar al título de Magíster en Tecnología Informática Aplicada en Educación no publicado. La Plata, Buenos Aires: Universidad Nacional de La Plata.

Pozo, J. (1998). Aprender y enseñar ciencia: Del conocimiento cotidiano al conocimiento científico. Madrid: Ediciones Morata.

Pozo, J. (2006). Aprenderyenseñar ciencia. Enfoques para la enseñanza de la ciencia. Madrid: Ediciones Morata.

Rayas, J. (2004). El reconocimiento de las ideas previas como condición necesaria para mejorar las posibilidades de los alumnos en los procesos educativos en ciencias naturales. Recuperado en mayo de 2013 de: http://unidad094centro.servehttp.com:90/upnnaturared/libros/Libro1.pdf. 
FORMATO DE IDEAS PREVIAS

UNIVERSIDAD DISTRITAL FRANCISCO JOSÉ DE CALDAS FACULTAD DE CIENCIAS Y EDUCACIÓN

PROYECTO CURRICULAR LICENCIATURA EN BIOLOGÍA ESPACIO ACADÉMICO: AULA EXPERIMENTAL

\section{INSTRUMENTO DE CARACTERIZACIÓN SOCIOCULTURAL DE LOS ESTUDIANTES DEL ATENEO JUAN EUDES DEL GRADO NOVENO}

Estudiantes:

La siguiente encuesta tiene como propósito conocer acerca de algunos de sus aspectos personales e intereses en el aprendizaje, los datos recogidos NO serán evaluados, únicamente serán utilizados en el diseño de las actividades que llevaremos en las próximas clases. Además de las preguntas, al final encontrarán un espacio para sugerencias y observaciones si desean aportarlas. De ante mano muchas gracias por su colaboración.

\section{Aspectos personales.}

- Nombre completo:

- Edad:

- Género: Femenino Masculino

- Estrato socioeconómico:

- Con que familiares vive: __Padresy hermanos __Abuelos__Tíos__otros

- Lugar de nacimiento:

- Hace cuanto vive en Bogotá:

\section{CONDICIONES ACADÉMICAS Y SOCIOECONÓMICAS.}

- ¿Cuánto tiempo tarda de su casa al colegio?:

- ¿Cuáles herramientas utiliza para realizar sus tareas?

- Libros de la casa

- Computador/internet

- Consulta a padres

- Ayuda de hermanos mayores

- Asistir alguna biblioteca

- Otras 
- ¿Cuál es la asignatura que más le gusta de su colegio?

- ¿Cuál es la asignatura que menos le gusta de su colegio?

- ¿Qué quiere estudiar o hacer después de culminar sus estudios en el bachillerato?

- Trabajar ¿En Qué?

- Estudiar ¿Qué carrera?

- Otra ¿Cuál?

\section{PREGUNTAS ESPECÍFICAS DE BIOLOGIA:}

De los animales que conoce de su entorno qué diferencias anatómicas distingue entre estos:

¿Cuál cree usted que es la importancia de la evolución en su vida?

¿Considera usted que es la especie más desarrollada? ¿Por qué?

Escriba un animal que conozca dentro de las siguientes clasificaciones:

- Filum Poríferos:

- FilumCnidarios:

- Filum Platelmintos:

- FilumNemátodos:

- Filum Anélidos:

- Filum Moluscos:

- Filum Artrópodos:

- Filum Equinodermos:

- Filum Cordados:

- Clase Peces:

- Clases Anfíbios:

- Clase Reptiles:

- Clase Mamíferos: 


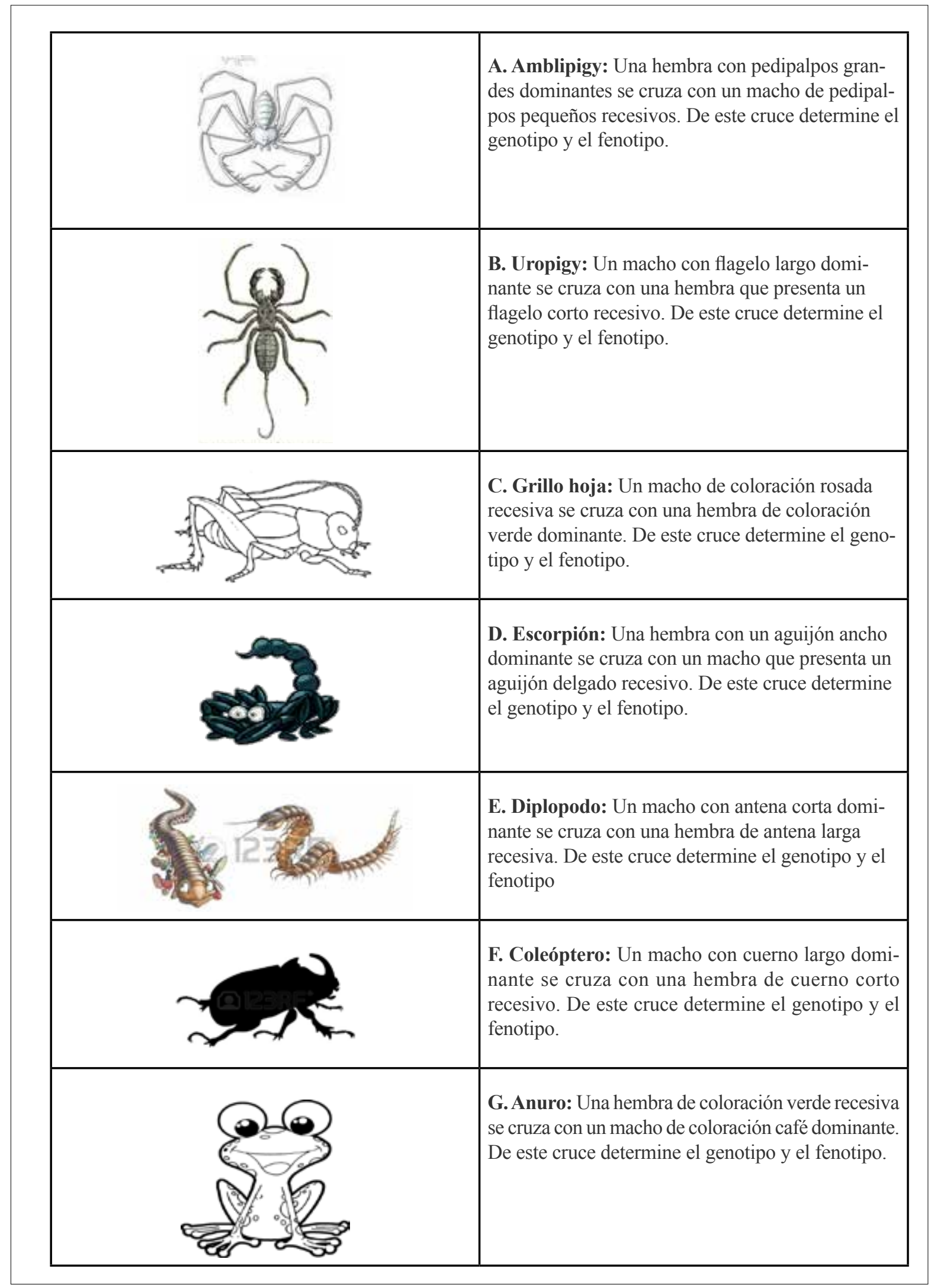


ANEXO NO. 3: FORMATO DE EVALUACION CRUCES MONOHIBRIDOS

\section{UNIVERSIDAD DISTRITAL FRANCISCO JOSÉ DE CALDAS ATENEO JUAN EUDES}

GRADO NOVENO

Primera evaluación: cruces monohíbridos

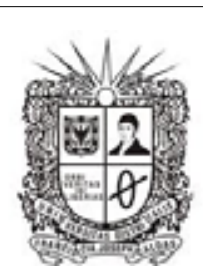

Nombre:

\begin{tabular}{|c|c|c|c|}
\hline \multicolumn{2}{|c|}{ EJEMPLOS DE CARACTERES DOMINANTES Y RECESIVOS EN LOS SERES HUMANOS } \\
\hline \multicolumn{2}{|c|}{ CARACTERES TRIVIALES } & \multicolumn{2}{c|}{ ENFERMEDADES O MALFORMACIONES } \\
\hline DOMINANTES & RECESIVOS & DOMINANTES & RECESIVOS \\
\hline Lengua enrollable & Lengua no enrollable & Enanismo & Estatura normal \\
\hline Rh+ & Rh - & Braquidactilia & Dedos normales \\
\hline Pelo rizado & Pelo liso & Corea de Huntington & Sin Corea de Huntington \\
\hline Cabello oscuro & Cabello claro & Pigmentación normal & Albinismo \\
\hline Ojos oscuros & Ojos claros & Coagulación normal de la sangre & Hemofilia \\
\hline Labios gruesos & Labios finos & Visión normal & Daltonismo \\
\hline Pestañas largas & Pestañas cortas & Oído normal & Sordomudez \\
\hline Oreja con lóbulo & Oreja sin lóbulo & Polidactilia & N. ${ }^{\circ}$ normal de dedos \\
\hline Grupos sanguíneos Ay B & Grupo sanguíneo O & Visión normal & Ceguera para los colores \\
\hline
\end{tabular}

Teniendo en cuenta los datos suministrados por la tabla No.1, desarrolle los siguientes ejercicios.

A. Un hombre con alelos dominantes para la característica de poder enrollar la lengua, se casa con una mujer que no puede hacerlo. Los alelos están representados por las letras (LL) para el dominante y (II) para el recesivo. ¿Qué fenotipo y genotipo se esperaría de esta unión en su descendencia?

B. Una mujer con cabello rubio espera un hijo de un hombre de cabello negro, teniendo en cuenta que la característica está representada por la letra C. ¿cómo se esperaría que naciera el bebé?

C. Un hombre que presenta la enfermedad de albinismos, se casa con una mujer homocigota dominante que no presenta esta enfermedad. ¿Cómo serán sus hijos? ¿presentarán la enfermedad?

D. Una mujer sordomuda se casa con un hombre que escucha normalmente. De esta unión se podría inferir que los hijos ¿presentan o no la enfermedad? 
ANEXO NO. 4: FORMATO DE EVALUACION CRUCES DIHIBRIDOS

\section{UNIVERSIDAD DISTRITAL FRANCISCO JOSÉ DE CALDAS ATENEO JUAN EUDES}

GRADO NOVENO

Segunda evaluación: ejercicios dihíbridos

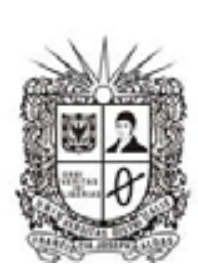

Nombre:

1. Se cruza una planta de tallos altos (Dominantes, TT) y flores violetas (Dominante, VV) homocigotas para ambos caracteres con una planta de tallos enanos (Recesivo, $\mathrm{tt}$ ) y flores (Recesivo, vv) blancas. A partir de esto determinar el genotipo y el fenotipo para su descendencia.

2. Un ratón hembra de color café (Dominante, CC) y cola larga (Dominante, LL) se cruza con un macho de color blanco (Recesivo, cc) y cola corta (Recesivo, II). A partir de esto determinar el genotipo y el fenotipo para su descendencia.

3. Un hombre de piel morena (Dominante, AA) y cabello crespo (Dominante, CC) se cruza con una hembra (Recesivo, aa) y cabello liso (Recesivo, cc). A partir de esto determinar el genotipo y el fenotipo para su descendencia.

4. Un caballo macho de crin largo (Dominante, $L L)$ y pelaje negro (Dominante, NN) se cruza con una yegua de crin corto (Recesivo, Il) y pelaje blanco (recesivo, nn). A partir de esto determinar el genotipo y el fenotipo para su descendencia. 